\title{
Remaining Useful Life Prognosis of Aircraft Brakes
}

\author{
Athanasios Oikonomou ${ }^{1 *}$, Nick Eleftheroglou ${ }^{2 *}$, Floris Freeman ${ }^{3}$, Theodoros Loutas ${ }^{4+}$, Dimitrios Zarouchas $^{5+}$ \\ ${ }^{1,4}$ Department of Mechanical Engineering and Aeronautics, University of Patras, Patras GR-26500, Greece \\ thanos.k.oik@gmail.com,thloutas@upatras.gr \\ ${ }^{2,5}$ Faculty of Aerospace Engineering, Delft University of Technology, Kluyverweg 1, 2629HS, Delft, the Netherlands \\ eleutherogloun@gmail.com,D.Zarouchas@tudelft.nl \\ ${ }^{3}$ KLM Royal Dutch Airlines, Schiphol, 1117 ZL, The Netherlands \\ Floris.Freeman@klm.com \\ *Co-first authors: These authors contributed equally to this work; ${ }^{+}$corresponding authors: T. Loutas, D. Zarouchas
}

\begin{abstract}
We investigate the performance of three different datadriven prognostic methodologies towards the Remaining Useful Life estimation of commercial aircraft brakes being continuously monitored for wear. The first approach utilizes a probabilistic multi-state deterioration mathematical model i.e., a Hidden Semi Markov model whilst the second utilizes a nonlinear regression approach through classical Artificial Neural Networks in a Bootstrap fashion in order to obtain prediction intervals to accompany the mean remaining life estimates. The third approach attempts to leverage the highly linear degradation data over time and uses a simple linear regression in a Bayesian framework. All methodologies, when properly trained with historical degradation data, achieve excellent performance in terms of early and accurate prediction of the remaining useful flights that the monitored set of brakes can safely serve. The paper presents a real-world application where it is demonstrated that even in non-complex linear degradation data the inherent data stochasticity prohibits the use of a simple mathematical approaches and asks for methodologies with uncertainty quantification.
\end{abstract}

\section{INTRODUCTION}

Aircraft maintenance ensures the airworthiness of the fleet by preventively maintaining aircraft systems and structures that are critical to safe and economic operations, and by correctively maintaining systems and structures that are not

\footnotetext{
Athanasios Oikonomou et al. This is an open-access article distributed under the terms of the Creative Commons Attribution 3.0 United States License, which permits unrestricted use, distribution, and reproduction in any medium, provided the original author and source are credited.

https://doi.org/10.36001/IJPHM.2022.v13i1.3072
}

critical. Time-based maintenance (TBM) is the current preventive practice for most of the aircraft components; they are inspected and repaired if needed, based on fixed intervals which are determined by flight hours, flight cycles or calendar days, whichever comes first. Interval lengths may vary from one cycle during pre-flight inspections to several years during complete aircraft overhaul. Frequent maintenance tasks increase the operational costs and the downtime of an aircraft. Most inspections do not lead to any required follow-up maintenance and could therefore have been omitted if the state of the aircraft had been known apriori.

An alternative practice to TBM would be to execute maintenance based on the real time health status of the aircraft, the so-called condition-based maintenance (CBM). $\mathrm{CBM}$ is a paradigm swift aiming to reliably assess the condition of the aircraft's systems and structures, confidently estimate the future health state and informatively support the operators for the decision making on when maintenance should be performed (Lee \& Mitici, 2020), (Kallen \& Noortwijk, 2005), (Li, Verhagen \& Curran, 2020), (Ezhilarasu, Skaf \& Jennions). The Advisory Council for Aeronautical Research in Europe (ACARE) envisages that, by 2050, all new aircraft will be designed for CBM and it is expected that CBM will contribute to a significant reduction in maintenance, repair and overhaul process time (ACARE, 2005). To put CBM in practice though, there is a need for assessing the current health state of a component and estimating its future condition and remaining useful life (RUL) in real-time ( $\mathrm{Li}$, Verhagen \& Curran, 2020), (Adhikari \& Buderath, 2016). The latter falls into the research field of prognostics; in particular, prognostics aim to provide reliable predictions and confidence to the operators for decision making that will 
convert health related information to values (Jia, Huang, Feng, Cai \& Lee, 2018).

In modern aircrafts, such as the BOEING 787 Dreamliner and AIRBUS A350, thousands of sensors are integrated within several systems, which record condition and health parameters during the operational life of the fleet. One of these sensorized systems are the aircraft's brakes. The brake system considered in this study is an electrically actuated carbon disc brake system embedded in each of the 8 wheels in the main landing gear of a wide-body aircraft. When activated, four brake actuators on each brake create a clamping force against the carbon-disc assembly, which creates friction and eventually decelerates the aircraft. Regular use of the brakes wears the pads and reduces their thickness. Two wear pins per brake system act as a visual indicator of the carbon thickness left. The aircraft itself measures the position of the actuators when clamped to the carbon discs and infers the carbon thickness from this measurement. This thickness can be wirelessly transmitted (as a percentage of original thickness) to the operator over ACARS (Aircraft Communications Addressing and Reporting System). A desirable thickness should be always present to ensure that the brakes are in a condition to stop the aircraft properly and are easily refurbished after removal.

Currently, the maintenance of brakes is performed under TBM. More specifically, two maintenance tasks are used; a manual visual inspection of the brake wear pins by a ground engineer at a fixed flight-cycle interval and the subsequent replacement if needed. If a certain amount of wear is observed, a pad replacement is scheduled but due to safety reasons and regulations, the interval of inspection is much shorter than the expected life cycle of the pad. As a result, only a fraction of the inspections results in a requirement for pad replacement. Real-time and remote estimation of the brakes' (future) condition would eliminate the need for these manual inspections, leading to a reduction in maintenance time. The electrical brakes could be one of the first examples of an aircraft system where a TBM policy may be substituted by CBM. The reason behind that is that the realtime monitoring health parameter (pad thickness) is very similar to the critical parameter that is manually inspected today. Hence, the use-case presented in this paper can help mature CBM in aircraft maintenance.

\section{Prognostics in AircRAft SyStems AND STRUCTURES}

Prognostics, and specifically RUL estimations, have been in the epicenter of research and development for more than a decade resulting in two main categories of methodologies (Goebel, Daigle, Saxena, Sankararaman, Roychoudhury \& Celaya, 2017); model-based prognostics (Autin, De Martin, Jacazio, Socheleau \& Vachtsevanos, 2021), (Acuna \& Orchard, 2016), (Dalla Vedova, Germanà, Berri \&
Maggiore, 2019) and data-driven prognostics (Rengasamy, Jafari, Rothwell, Chen \& Figueredo, 2020), (Verstraete, Droguett \& Modarres, 2020). In the field of aircraft systems prognostics, few works have been published the last 10 years with most of them dealing with the famous C-MAPPS simulation dataset from turbofan engines. In Autin et al. (2021), a model-based prognostic methodology that utilizes a high-fidelity dynamical model of flight control servoactuators and particle-filtering has proven very efficient in fault detection and failure prognosis. Particle-filtering-based prognostics has been indeed a popular approach in modelbased prognostics and gives excellent predictions when a physical model exists. In Dalla Vedova et al. (2019), the authors proposed a model-based fault detection and isolation method, employing a Genetic Algorithm (GA) to identify failure precursors before the performance of the system starts being compromised. In the data-driven field, we can indicatively mention (Rengasamy et al., 2020), (Verstraete et al. 2020), (Che, Wang, Fu, \& Ni, 2019) (Lu, Wu, Huang $\&$ Qiu, 2019) where deep learning or logistic regression approaches have been successfully implemented for aircraft turbofan engine failure prognostics on simulated data. Both data-driven and model-based methodologies have their merit in the successful implementation of prognostics and their employment should be done considering two factors; the existence of a physical/phenomenological model that describes the degradation process and the availability and quality of condition or the existence of historical health monitoring degradation data under the various health states. While model-based methodologies are considered to be more accurate as they capture the physical phenomenon and they are easier to be understood by the operator/user, datadriven methodologies become very popular nowadays as they can be scaled to multiple systems without the need for specific domain knowledge. The availability of vast amount of data, the increase of computational power and the capability of statistical models and/or Artificial Intelligence (AI) algorithms to use and learn from real world degradation data and train algorithms for reliable RUL estimations, constitute the data-driven approaches a cost-effective alternative to physics-based modelling (Dawn, Kim \& Choi, 2015).

Data Analytics offer a wide range of mathematical algorithms which can be employed in a prognostic framework for RUL estimations; among them are artificial neural networks, i.e. deep learning, LSTM and Bayesian versions, logistic and Gaussian regression processes, Hidden Markov models (Loutas, Eleftheroglou \& Zarouchas, 2017), (Eleftheroglou, Zarouchas, Loutas, Alderliesten \& Benedictus, 2018) have been utilized for developing datadriven prognostics frameworks and demonstrating their capabilities for aircraft systems as well as aircraft materials and structures. There is no common rule for the selection of an algorithm and it mainly depends on the knowledge about the system's operational behavior, the associated historical 
data and the user's experience and skillfulness to apply a certain type of algorithm. Nevertheless, as the accuracy of estimations is conditional to uncertainties, such as incomplete knowledge of the future loading and environmental conditions, noisy or faulty data and the use of inaccurate models, it is essential that the algorithms can express a confidence about their prediction. When designing the prognostics framework, if uncertainty is not considered or carefully interpreted, the predictions could be meaningless compromising the mission of prognostics (Sankararaman \& Goebel, 2015).

The contribution of the present paper is to assess the feasibility of real-time and remote RUL prognostication via probabilistic data-driven methodologies in a new real-life degradation dataset from aircraft brakes. A real-world application is presented where we demonstrate that even in non-complex linear degradation data the inherent data stochasticity prohibits the use of a simple mathematical approaches and methodologies with uncertainty quantification are required. More specifically Artificial Neural Networks (ANN) with bootstrapping, a Bayesian approach to the classical Linear Regression (BLR) as well as the Non-Homogeneous Hidden Semi Markov Model (NHHSMM). ANN is a classical choice in regression problems and the prediction problem might as well be considered as such. The BLR is selected after observing the highly linear nature of the data. The NHHSMM is a statistical model more rich in structure and complex from a mathematical point of view and was found to outperform state-of-the-art machine learning algorithms in a series of studies that the authors published (Loutas, Eleftheroglou \& Zarouchas, 2017), (Eleftheroglou, Zarouchas, Loutas, Alderliesten \& Benedictus, 2018), (Eleftheroglou, Mansouri, Loutas, Karvelis, Georgoulas, Nikolakopoulos \& Zarouchas, 2019), (Loutas, Eleftheroglou, Georgoulas, Loukopoulos, Mba \& Bennett, 2020) thus is believed to be a challenging competitor to regression algorithms.

The remainder of the paper is organized as follows: Section 3 presents the dataset for the wear of the brake pads, the data pre-processing and how the training/test data separation was performed. Section 4 summarizes the basic principles of the 3 data-driven models. Section 5 presents and discusses the results for the RUL estimations while section 6 compares the performance of the models using several performance metrics. The conclusions are given in section 7 , along with a discussion for future work.

\section{Methodology}

The methodology developed in the present work is summarized in Figure 1. It starts from a representative dataset with historical degradation data from aircraft brakes wear. These data are pre-processed to form appropriate input for the data-driven models training. After the training, the models may be fed with unseen field sensor data and offer predictions of the RUL. This is a critical input for the maintenance management and could serve a future transition for a periodic maintenance paradigm to a CBM one.

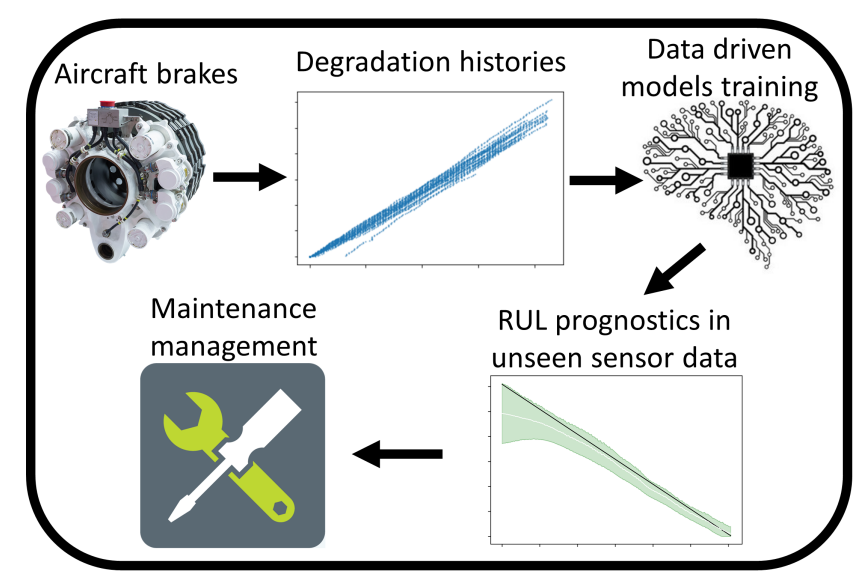

Figure 1. Concept and methodology flowchart

\subsection{Dataset}

A real-life historical dataset from the widebody fleet of a European airline is utilized. The dataset deals with the usage and degradation of the brakes of several aircraft in a widebody fleet. The brakes refer to the braking system of the main landing gear. Every aircraft has eight brakes. They have some built-in features, such as a continuous real-time measurement of the carbon disc thickness. Over time, due to wear, the thickness of the brake disc reduces. When the thickness of a brake's disc is thinner than a threshold, the brake is replaced within a limited number of aircraft cycles. In its initial form, the dataset consists of full registration histories for each plane, after each flight. Specifically, it consists of the landing date and time, the departure and landing airport, and eight columns with the real-time measurement of the carbon disc thickness left, expressed in percentage. The dataset needs to be transformed to a more usable form in order to be used as input into the prognostic algorithms.

\subsection{Data Preprocessing}

Each brake is assumed to be an independent system, its degradation history is independent of the other brakes' histories and no connection is considered between the brake's RUL and the airplane that it belongs to, or to the brake's position. So, for each brake a full dataset consisting of three columns. Table 1 gives an example of how the final dataset is organized. The first column entails the already performed flights, the second column gathers the sensor indications regarding the percentage (\%) of remaining brake disc thickness, which can be calculated as: $\left(100-\right.$ wear $\left._{\text {carbon disc }}\right) \%$, and the last column represents the expected output; i.e., the remaining useful flights before 
replacement for each brake. We consider the performed flights variable as the only time variable because the brakes are only used during the landing phase of the flight. After creating complete brake degradation histories, a failure threshold is defined at the first time the sensor value reaches

\begin{tabular}{ccc}
\hline Performed Flights & Sensor_Data (\%) & RUL (flights) \\
\hline 1062 & 0 & 0 \\
\hline 1056 & 1 & 6 \\
\hline 1050 & 1.5 & 12 \\
\hline$\ldots$ & $\ldots$ & $\ldots$ \\
\hline 12 & 76 & 1050 \\
\hline 6 & 76.33 & 1056 \\
\hline 0 & 77.17 & 1062 \\
\hline
\end{tabular}

Table 1. Sensor data structure for an aircraft brake

zero, and we do not consider any flights performed from that point on, if any. This threshold accounts for a safety buffer; in reality, a non-zero pad thickness is left for safe operations, even when the sensor reading is at $0 \%$. Furthermore, a 6-flights moving average window is applied to smoothen the data as eq. (1) explains, with $i \in[0,6)$ and $k \in[1,7,13, \ldots]$. The correlation between those two input variables (performed flights and sensor data) and the expected output (Remaining Useful Life) is depicted in Figure 2.

$$
\begin{aligned}
& \text { Flights_Performed }=\max (\text { Flights_Performed }(k+i)), \\
& \text { Sensor_data }=\text { average }(\text { Sensor_data }(k+i)), \\
& R U L=\min (R U L(k+i)),
\end{aligned}
$$

\subsection{Training/Testing datasets preparation}

Out of the total 56 available brake degradation histories, 27 histories are selected to be used as the dataset for training, evaluating and testing the methods chosen for predicting the remaining useful life. The remaining 29 are considered incomplete degradation histories and therefore are not included. Those 27 are selected as they are complete degradation histories i.e., disc brake pad thickness decays from $100 \%$ to $0 \%$. 3 out of 27 exhibit a rather different degradation rate, as compared to the rest, and are considered as outliers. Two of them are left outliers, since their lifespans are shorter than the rest of the population on average. The third is a right outlier i.e., it has a longer lifespan. The outliers are used only in the testing phase to check the proposed algorithms' predictive capabilities, since one prognostic challenge is to be able to predict accurately the RUL of an outlier without using it in the training process. The remaining 24 histories represent complete sensor data from 8 brake sets of 3 aircrafts. For the training of the prognostic algorithms a k-fold leave-two-out method was used. Randomly, one of those 3 aircrafts is chosen to provide the testing and validation set each time. Hence, 16 out of the 24 brakes constitute the training set at all times.

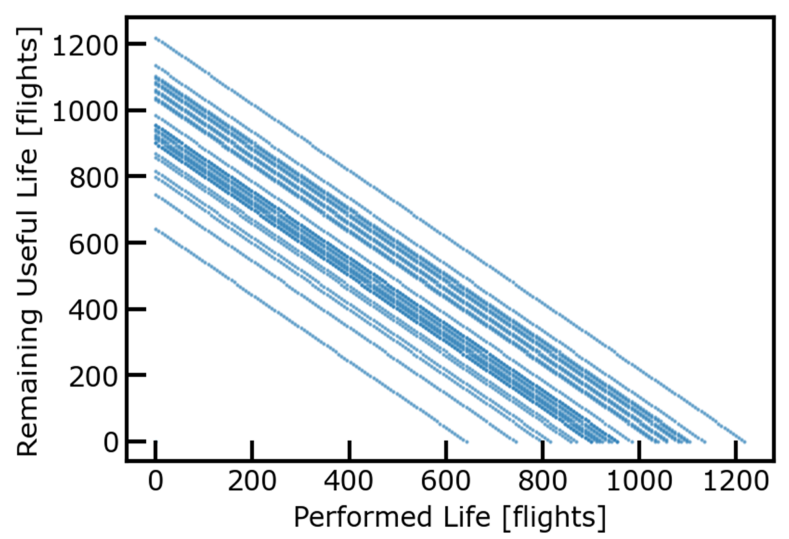

(a)

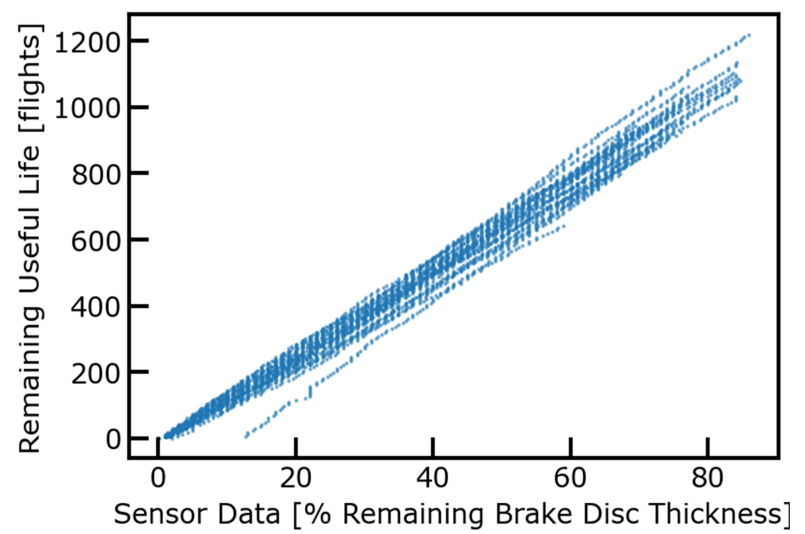

(b)

Figure 2. A representative (partial) overview of the brake degradation dataset a) The correlation between RUL and already performed flights $b$ ) The correlation between RUL and the sensor data.

Then, the following process is repeated 8 times. Out of the remaining 8 brakes, for each run, one is picked as the test set, the following one as the validation, and then the remaining six of them are placed back at the training set. The procedure is summarized in Figure 3.

\section{MAthematical Models for Prognostics}

Three different mathematical models are selected based on previous experience of the authors, to deal with the estimation of the RUL as well as the uncertainty quantification i.e., determination of confidence intervals. First, after observing the rather linear degradation behavior of sensor data in Figure 2, linear regression with a Bayesian implementation to quantify uncertainty is suggested. Second option is a Generalized Hidden Semi Markov model that considers degradation as a multi-state process, a latent Markov chain which manifests itself through condition monitoring data. Finally, an Artificial Neural Network 
(ANN) is utilized through a bootstrapping approach to determine confidence intervals to the mean predictions.

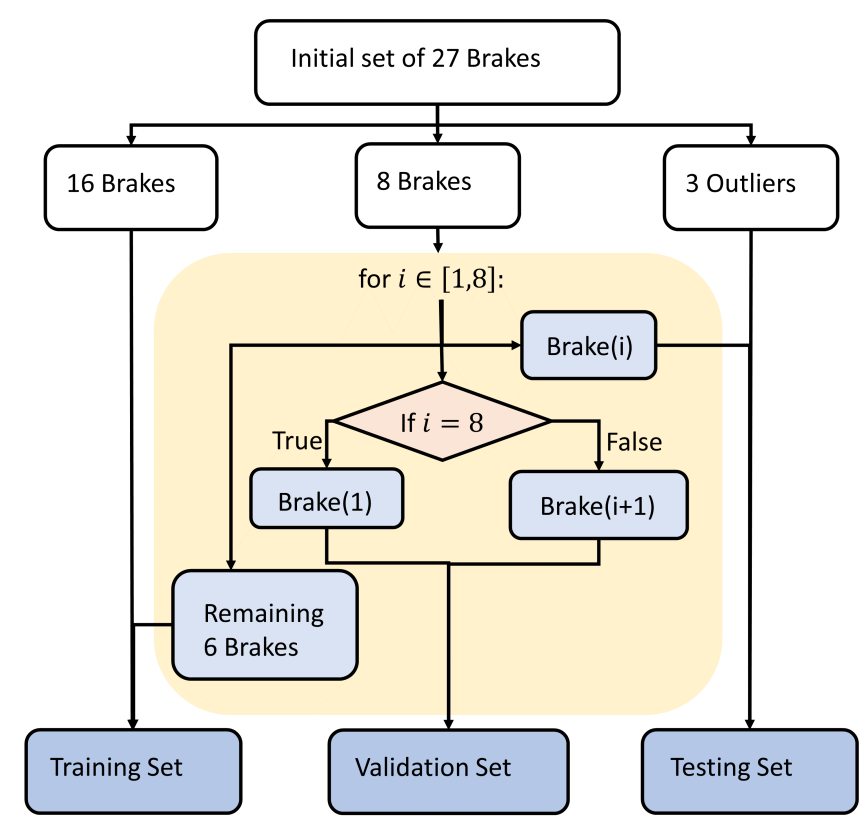

Figure 3. The process for choosing Test and Validation Set each time

\subsection{Bayesian Linear Regression (BLR)}

In linear regression a target $t$ is approximated by a linear function $\mathrm{y}\left(\mathrm{x}_{\mathrm{i}}, \mathbf{w}\right)=\mathbf{w}^{\mathrm{T}} \mathbf{x}$ as follows $\mathrm{t}_{\mathrm{i}}=\mathrm{y}\left(\mathrm{x}_{\mathrm{i}}, \mathbf{w}\right)+\varepsilon_{\mathrm{i}}$ where $\varepsilon_{\mathrm{i}} \sim \mathrm{N}\left(0, \sigma^{2}\right)$. The Bayesian perspective on classical linear regression does not consider the model's weights as deterministic but instead considers them random variables and sets Gaussian priors with zero mean and potentially variable variance at each weight i.e. $p(\mathbf{w} \mid \boldsymbol{\alpha})=$ $\prod_{i=0}^{\mathrm{N}} \mathrm{N}\left(\mathrm{w}_{\mathrm{i}} \mid 0, \alpha_{\mathrm{i}}^{-1}\right)$. In a fully Bayesian approach the model's hyperparameters are also random variables defined by Gamma distributions i.e., $\mathrm{p}(\boldsymbol{\alpha})=\prod_{\mathrm{i}=0}^{\mathrm{N}} \operatorname{Gamma}\left(\alpha_{\mathrm{i}} \mid \mathrm{a}, \mathrm{b}\right)$ and $\mathrm{p}\left(\sigma^{2}\right)=\operatorname{Gamma}\left(\sigma^{2} \mid \mathrm{c}, \mathrm{d}\right)$. The parameters a,b,c,d of the Gamma hyperpriors are fixed to $10^{-6}$. The parameters $\boldsymbol{\alpha}$ and $\sigma^{2}$ are estimated iteratively by maximizing the log marginal likelihood of the targets $\mathrm{p}\left(\mathrm{t} \mid \boldsymbol{\alpha}, \sigma^{2}\right)$ which comes in closed form. The scikit-learn 0.23.2 python software was utilized for the implementation. This is based on the algorithm described in (Tipping, 2001) where all the necessary mathematical details are given.

\subsection{Non-Homogenous Hidden Semi Markov Model (NHHSMM)}

NHHSMM is a mathematical model that describes the association between a hidden stochastic degradation process and an observed one which manifests via condition/health monitoring data. The NHHSMM is actually a double stochastic process. The hidden process is a finite Semi Markov chain and the observed process, conditioned on the hidden one. To properly describe the bi-dimensional stochastic process, the model's parameters $\boldsymbol{\theta}$ need to be estimated. These parameters characterize the transition rate distribution between the hidden states (degradation process), as well as the correlation between the hidden states and historical data (observation process). The studied system is assumed to start its operation from a healthy state and during its service life transits to states of higher degradation until it reaches its failure state.

The model's parameters $\boldsymbol{\theta}$ are obtained via a Maximum Likelihood Estimator (MLE) $\boldsymbol{\theta}^{*}$ of the model parameters $\boldsymbol{\theta}$ through a procedure described in detail in (Moghaddass \& Zuo, 2014). The MLE algorithm leads to the maximization the likelihood function $\mathrm{L}\left(\boldsymbol{\theta}, \mathbf{x}^{(1: \mathrm{M})}\right)$ Eq. (2), where $\mathbf{x}^{(\mathrm{m})}$ is the $\mathrm{m}$-th degradation history, $\mathrm{M}$ is the number of available degradation histories.

$$
\begin{aligned}
& \mathrm{L}\left(\boldsymbol{\theta}, \mathbf{x}^{(1: \mathrm{M})}\right)=\prod_{\mathrm{m}=1}^{\mathrm{M}} \operatorname{Pr}\left(\mathbf{x}^{(\mathrm{m})} \mid \boldsymbol{\theta}\right) \\
& \stackrel{\mathrm{L}^{\prime}=\log (\mathrm{L})}{=} \mathrm{L}^{\prime}\left(\boldsymbol{\theta}, \mathbf{x}^{(1: \mathrm{M})}\right)=\sum_{\mathrm{m}=1}^{\mathrm{M}} \log \left(\operatorname{Pr}\left(\mathbf{x}^{(\mathrm{m})} \mid \boldsymbol{\theta}\right)\right) \\
& \Rightarrow \boldsymbol{\theta}^{*}=\arg \max _{\boldsymbol{\theta}}\left(\sum_{\mathrm{m}=1}^{\mathrm{M}} \log \left(\operatorname{Pr}\left(\mathbf{x}^{(\mathrm{m})} \mid \boldsymbol{\theta}\right)\right)\right)
\end{aligned}
$$

Initializing with random values for $\boldsymbol{\theta}$ and solving the aforementioned optimization problem the parameter estimation process is concluded and prognostic-related measures can be defined and calculated. The mean value and the associated confidence intervals of RUL are obtained through the conditional reliability function $\mathrm{R}\left(\mathrm{t} \mid \mathrm{x}_{1: \mathrm{t}_{\mathrm{p}}}, \mathrm{L}>\mathrm{t}_{\mathrm{p}}, \boldsymbol{\theta}^{*}\right)=\operatorname{Pr}\left(\mathrm{L}>\mathrm{t} \mid \mathrm{x}_{1: \mathrm{t}_{\mathrm{p}}}, \mathrm{L}>\mathrm{t}_{\mathrm{p}}, \boldsymbol{\theta}^{*}\right)$ i.e. the probability that the studied system continues its safe operation after a time point $t$ (less than a considered lifetime limit $L$ ) further than the present time $t_{p}$, given the optimal model parameters $\boldsymbol{\theta}^{*}$. This is a definition apparently conditional on available historical health data (the observation sequence $x_{1: t_{p}}$ ) and the estimated model's parameters $\boldsymbol{\theta}^{*}$. The mean RUL is obtained as the integral of the conditional reliability as shown in Eq. (3) whereas the confidence intervals are calculated through the cumulative distribution function (CDF) of RUL in Eq. (4).

$\operatorname{RUL}\left(\mathrm{t} \mid \mathrm{x}_{1: \mathrm{t}_{\mathrm{p}}}, \mathrm{L}>\mathrm{t}_{\mathrm{p}}, \boldsymbol{\theta}^{*}\right)=\int_{0}^{\infty} \mathrm{R}\left(\mathrm{t}+\tau \mid \mathrm{x}_{1: \mathrm{t}_{\mathrm{p}}}, \mathrm{L}>\mathrm{t}_{\mathrm{p}}, \boldsymbol{\theta}^{*}\right) \mathrm{d} \tau$
$\operatorname{Pr}\left(\mathrm{RUL}_{\mathrm{t}_{\mathrm{p}}} \leq \mathrm{t} \mid \mathrm{x}_{1: \mathrm{t}_{\mathrm{p}}}, \boldsymbol{\theta}^{*}\right)=1-\mathrm{R}\left(\mathrm{t}+\tau \mid \mathrm{x}_{1: \mathrm{t}_{\mathrm{p}}}, \mathrm{L}>\mathrm{t}_{\mathrm{p}}, \boldsymbol{\theta}^{*}\right)$

\subsection{Bootstrapped Artificial Neural Networks (BNN)}

Artificial Neural Networks (ANN) are a well-known class of AI algorithms with regression and classification capabilities. They are highly capable of capturing linear and non-linear correlation between input data and expected output if provided with sufficient data. Considering a stochastic 
process, such as a system's degradation, the $i^{\text {th }}$ measured target $t_{i}$ can be represented as:

$$
\mathrm{t}_{\mathrm{i}}=\mathrm{y}\left(\mathrm{x}_{\mathrm{i}}\right)+\varepsilon_{\mathrm{i}}=\mathrm{f}\left(\mathrm{x}_{\mathrm{i}}, \mathbf{w}\right)+\varepsilon_{\mathrm{i}}
$$

where $\mathrm{y}\left(\mathrm{x}_{\mathrm{i}}\right)$ is the real regression mean and $\varepsilon_{\mathrm{i}}$ is a random variable with zero expectation, representing some noise. $f\left(x_{i}, \mathbf{w}\right)$ is a function trying to correlate the input variables $x_{i}$ with the true regression mean $y\left(x_{i}\right)$ given a set of variables $\mathbf{w}$. The output of an ANN model $\mathrm{y}_{\text {pred }}\left(\mathrm{x}_{\mathrm{i}}\right)$ is an estimate of the true regression mean $\mathrm{y}\left(\mathrm{x}_{\mathrm{i}}\right)$ :

$\mathrm{y}_{\text {pred }}\left(\mathrm{x}_{\mathrm{i}}\right)=\mathrm{f}\left(\mathrm{x}_{\mathrm{i}}, \mathbf{w}\right)$

And the prediction error can be expressed as:

$\mathrm{t}_{\mathrm{i}}-\mathrm{y}_{\text {pred }}\left(\mathrm{x}_{\mathrm{i}}\right)=\left[\mathrm{y}\left(\mathrm{x}_{\mathrm{i}}\right)-\mathrm{f}\left(\mathrm{x}_{\mathrm{i}}, \mathbf{w}\right)\right]+\varepsilon_{\mathrm{i}}$

There are two types affecting the prediction error $\varepsilon_{\mathrm{i}}$ in eq.7, the epistemic and the aleatoric error. Assuming statistical independence between the two, the total variance can be expressed as $\sigma_{\mathrm{t}}^{2}\left(\mathrm{x}_{\mathrm{i}}\right)=\sigma_{\mathrm{y}_{\text {pred }}}^{2}\left(\mathrm{x}_{\mathrm{i}}\right)+\sigma_{\varepsilon}^{2}\left(\mathrm{x}_{\mathrm{i}}\right)$. The variance of the epistemic error comes from the model mis-specification uncertainty. The variance of the aleatoric error comes from the inherent noise in the real-world data. Bootstrapping is a meta-algorithm, a method for constructing confidence intervals using the outcomes of several AI models with deterministic outputs such as the ANNs. It is a data resampling technique that aims at approximating the unknown statistical distribution of the error by an empirical distribution (Efron \& Tibshirani, 1993).

As Figure 4 summarizes, given a dataset, B ANN models are randomly initialized and the dataset splits into B training sets by resampling with replacement. We train those B ANNs using as a cost function the mean squared error. Confidence Intervals can be constructed if we realize a large number of ANNs $(\mathrm{B}>100)$ and assume that the predictions follow a normal distribution as utilized in (Khosravi, Nahavandi \& Atiya, 2011), (Pierce, Worden \& Bezazi, 2008), (El-Sayed, Riad, Elsafty \& Estaitia, 2017). The mean prediction values and the variance can then be simply calculated as:

$$
\begin{aligned}
& \mathrm{y}_{\text {mean }}\left(\mathrm{x}_{\mathrm{i}}\right)=\frac{1}{\mathrm{~B}} \sum_{\mathrm{b}=1}^{\mathrm{B}} \mathrm{y}_{\text {pred }}\left(\mathrm{x}_{\mathrm{i}}\right) \\
& \sigma_{\mathrm{y}_{\text {pred }}}^{2}\left(\mathrm{x}_{\mathrm{i}}\right)=\frac{1}{\mathrm{~B}-1} \sum_{\mathrm{b}=1}^{\mathrm{B}}\left(\mathrm{y}_{\text {mean }}\left(\mathrm{x}_{\mathrm{i}}\right)-\mathrm{y}_{\text {pred }}\left(\mathrm{x}_{\mathrm{i}}\right)\right)^{2}
\end{aligned}
$$

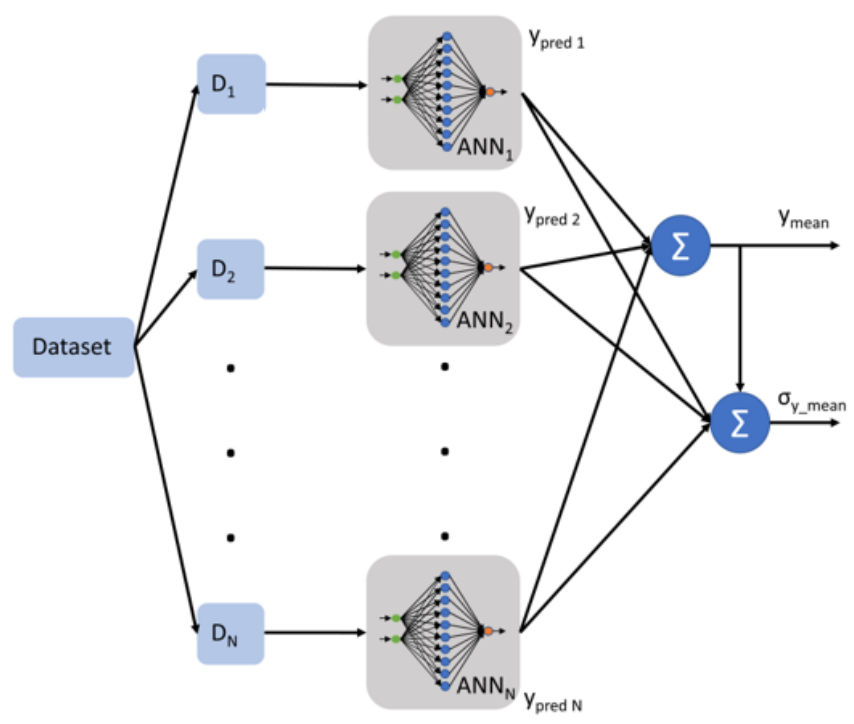

Figure 4. Bootstrapping ANN for uncertainty quantification

The variance $\sigma_{\mathrm{y}_{\mathrm{pred}}}^{2}$ comes mainly from random initialization of the model parameters and the use of different datasets for training the ANNs. Once the mean value and variance are estimated, the $\mathrm{i}^{\text {th }} \mathrm{CI}$ with a confidence level of $(1-\mathrm{a}) \%$ can be constructed as:

$\mathrm{y}_{\text {mean }}\left(\mathrm{x}_{\mathrm{i}}\right) \pm \mathrm{t}_{1-\frac{\mathrm{a}}{2}} \sqrt{\sigma_{\mathrm{y}_{\text {pred }}}^{2}\left(\mathrm{x}_{\mathrm{i}}\right)}$

In the present study, 200 ANNs were realized for the method. Each $\mathrm{ANN}_{\mathrm{y}}$ has one hidden layer with twenty neurons. The activation function is ReLU, except for the output neuron which has a linear activation function and the data are normalized in the $[-1,1]$ range. The learning rate was set to $10^{-4}$ with a decay rate of $10^{-5}$. A gradient descent-based Adam optimizer was used.

\section{RESULTS AND DISCUSSION}

Mean RUL predictions and 95\% confidence intervals for six of the total eleven brakes that were used as test sets, are presented in Figure 5 and Figure 6. Brakes 3, 6 and 8 concern normal systems of similar behavior, while Brakes 911 are the aforementioned outliers i.e. the brakes that experience shorter or higher lifetime than the average. 


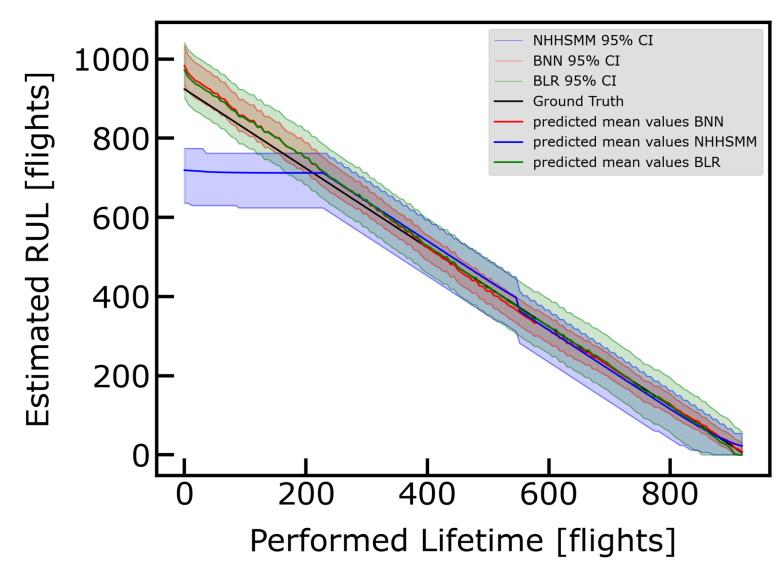

(a)

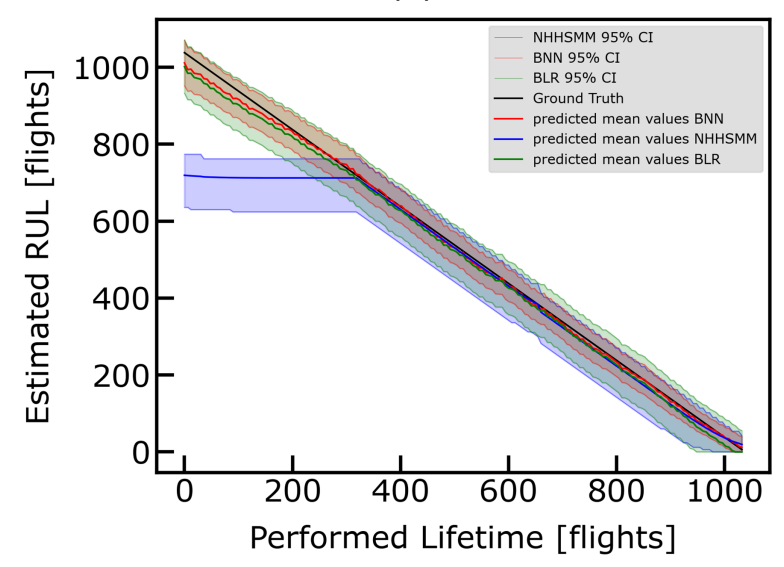

(b)

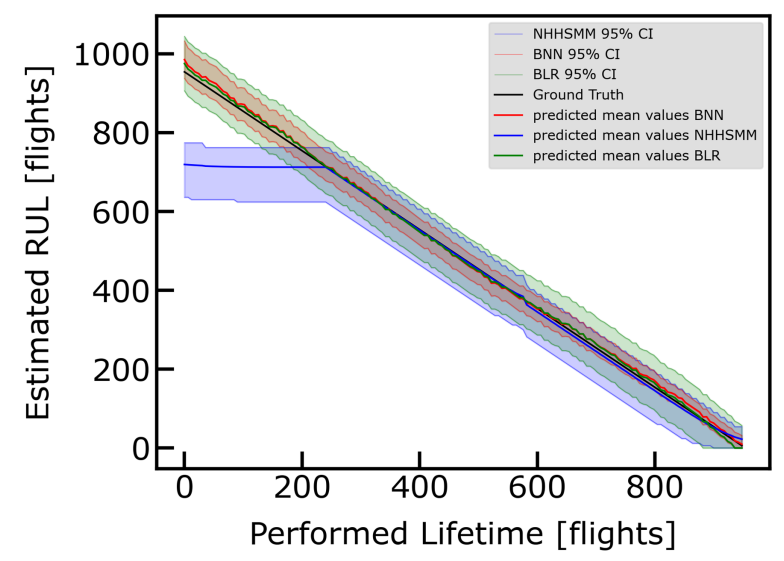

(c)

Figure 5. Prognostic Results with 95\% confidence intervals on (a) Brake \#3, (b) Brake \#6, (c) Brake \#8

Looking closely the results of Figure 5 we can see that at the beginning of the operational life, NHHSMM rather fails to capture the ground truth in all case studies mainly due to the selection of the number of possible discrete degradation states $(\mathrm{N})$. In the present study $\mathrm{N}=4$ is chosen since $\mathrm{N}>4$ will increase the computational effort without providing more reliable prognostics.

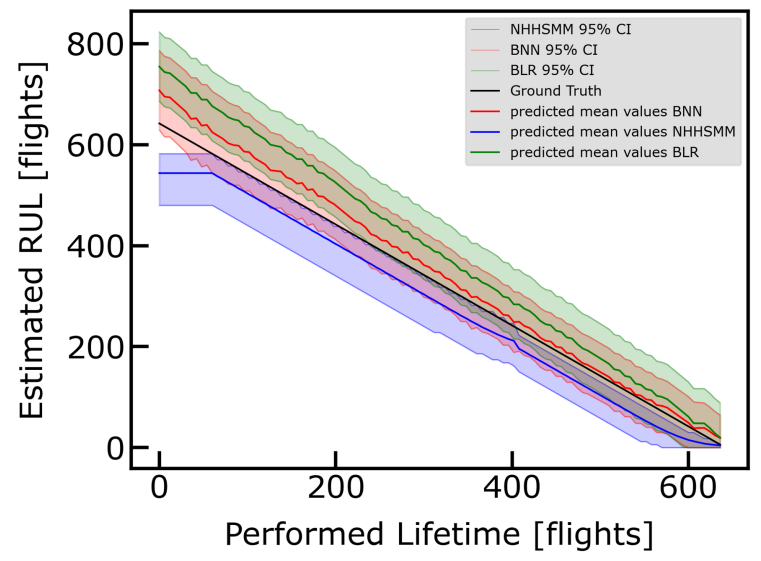

(a)

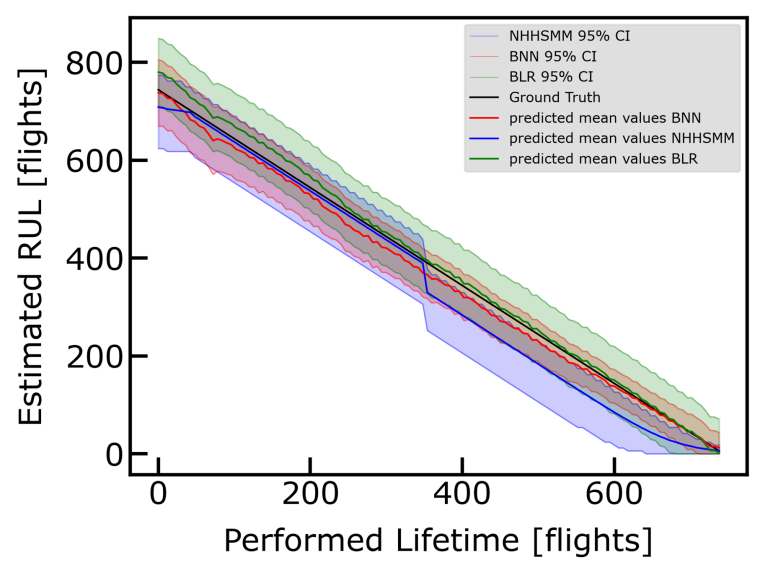

(b)

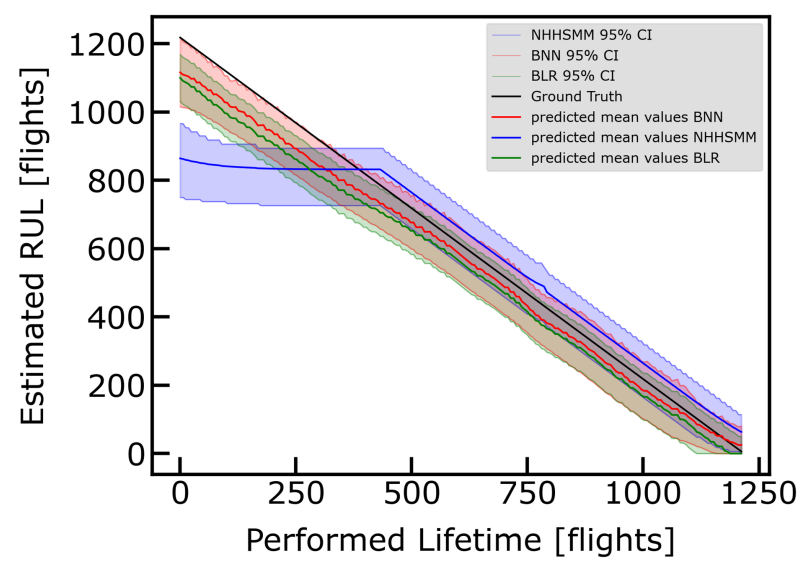

(c)

Figure 6. Prognostic Results with $95 \%$ confidence intervals on (a) Brake \#9 (Outlier 1), (b) Brake\#10 (Outlier 2), (c) Brake \#11 (Outlier 3)

It is generally admitted that early operation time RUL predictions cannot be reliable due to limited real-time data and possible future operation uncertainties. On the other hand, both BLR and BNN have no difficulty to follow the degradation trend from its beginning. It is obvious that in all presented case studies, BNN has the narrowest confidence 
interval, while BLR and NHHSMM have wider CIs. It is also worth mentioning that NHHSMM, for the majority of the presented results, provides more conservative mean estimates, while the mean estimates of BNN and BLR are, in most cases, close to each other. While NHHSMM, after overcoming the initial plateau, seems to have a clear monotonic tendency, both BLRs and BNNs mean estimates have some volatility. This volatility appears to be present at the same $\mathrm{x}$-values for both models, revealing the existence of some possible abnormalities in the dataset. It is highly notable that all three models mean predictions converge very close to the ground truth as the end of lifetime approaches and it is of paramount importance to have successful predictions. The width of confidence intervals decreases as well as operational time passes leading to increasingly more confident mean estimates.

Figure 6 summarizes the prognostic result for the outlier cases (Brake 9, Brake 10, Brake 11). Outliers as mentioned previously are systems that degrade sooner than average or later than average, and therefore experiencing shorter or higher lifetime than average. In our case Brake 9 and Brake 10 are left outliers, as they degrade sooner than average, whilst Brake 11 is considered as a right outlier since it degrades later than average. From the results shown in Figure 5 we can make the following comments. BNN outperforms NHHSMM and BLR for both left and right outliers, since ground truths seem to be within the predicted $\mathrm{CI}$ and the mean values seem to be close to ground truth even from the very beginning. The BNN estimated CIs are wider regarding the outliers, than the predicted CIs for the other eight brakes, while BLR and NHHSMM provide CIs of almost the same width. To quantify even more these qualitative observations, we proceed to a prognostic performance assessment through special metrics.

\section{Prognostic Performance Metrics}

The metrics used in our case study assess both the mean value predictions as well as Confidence Intervals (CI). For the assessment of the $\mathrm{CI}$, the most important characteristic is the coverage probability CICP (Confidence Interval Coverage Probability). It is measured by counting every target value that is in the defined confidence interval.

$C I C P=\frac{1}{n} \sum_{i=1}^{n} \xi_{i}\left(I_{a}\left(x_{i}\right), t_{i}\right)$,

where $\xi_{i}\left(I_{a}\left(x_{i}\right), t_{i}\right)= \begin{cases}1, & t_{i} \in I_{a}\left(x_{i}\right) \\ 0, & \text { otherwise }\end{cases}$

and where, $n$ is the number of target values that belong inside the confidence interval $I_{a}\left(x_{i}\right),(1-\mathrm{a}) \%$. Another crucial metric concerning the $\mathrm{CI}$ is the mean confidence interval width (MCIW), which can be easily expressed as:

$M C I W=\frac{1}{n} \sum_{i=1}^{n}\left(U_{i}-L_{i}\right)$ with $U_{i}$ and $L_{i}$ being the upper and lower value of the CI respectively. For the assessment of the predicted mean values several metrics as analyzed in the classical work of (Saxena, Celaya, Saha, Saha \& Goebel, 2010) are used. The Root Mean Squared Error (RMSE), the Mean Absolute Percentage Error (MAPE), the Prognostic Horizon (PH) and the Cumulative Relative Accuracy (CRA) are defined in the following:

$$
\begin{aligned}
& R M S E=\sqrt{\frac{\sum_{i=1}^{n}\left(E_{m}\left(t_{i}\right)\right)^{2}}{n}} \\
& M A P E=\frac{100}{n} \sum_{i=1}^{n} \frac{\left|E_{m}\left(t_{i}\right)\right|}{y_{\text {true }}\left(t_{i}\right)} \\
& P H=E O L-t_{i} \\
& C R A=\frac{\sum_{i=1}^{n} R A\left(t_{i}\right)}{N}, \text { where } R A\left(t_{i}\right)=1-\left|\frac{E_{m}\left(t_{i}\right)}{y_{\text {true }}\left(t_{i}\right)}\right| \\
& \text { and } E_{m}\left(t_{i}\right)=y_{\text {true }}\left(t_{i}\right)-y_{\text {mean }}\left(t_{i}\right)
\end{aligned}
$$

Besides RMSE and MAPE which are well known and widely used in prognostic results assessment, the Prognostic Horizon is the difference between a time $t_{i}$, when the predictions meet specified performance criteria, and the time corresponding to the end of life (EoL). Cumulative Relative Accuracy is the normalized sum of relative prediction accuracies at specific time instances. More details regarding the metrics can be found in the classical paper of Saxena et al. [30]. In Table 2 the prognostic performance metrics for all the brakes of the test set are presented.

Although predictions are available from the very onset of the operational phase of the brakes, we focus on the performance at the $75 \%$ of the lifetime and thus we calculate the metrics ignoring the first $25 \%$ of the lifetime of each brake. It is desirable for CICP and CRA to get the maximum value of 1 and for the $\mathrm{PH}$ a maximum value of 0.75 (since we focus on the performance at the $75 \%$ of the lifetime), while the rest of the presented metrics (MCIW, RMSE, MAPE) are desirable to take as low values as possible.

The average metric values across all eleven brakes are also calculated and presented in Table 2. Overall, the BNN clearly outperforms the other two models with BLR performing second best and NHHSMM being the worst of the three. More specifically, regarding RMSE and MAPE metrics, which represent the error of the predicted mean RUL from the ground truth RUL, BNN outperforms the other two methods in almost every single case. BLR(12) performs well in normal degradation scenarios, however, it fails to accurately predict the RUL of the outliers. Although NHHSMM performs quite well close to the brake's end of 
life, it fails to do so in the initial part, in almost every case, which leads to poor RMSE and MAPE metrics.

Table 2: Prognostic performance metrics for all brakes of the test set and average metric values (with bold in the bottom of the table the best average performance attained among the three algorithms)

\begin{tabular}{|c|c|c|c|c|c|c|c|}
\hline $\begin{array}{c}\text { Brake } \\
\#\end{array}$ & Algorithm & CICP & MCIW & RMSE & MAPE & CRA & $\mathrm{PH}$ \\
\hline \multirow{3}{*}{1} & BNN & 0.42 & 60.45 & 36.78 & 8.42 & 0.90 & 0.74 \\
\hline & NHHSMM & 0.55 & 125.50 & 47.57 & 11.10 & 0.85 & 0.74 \\
\hline & BLR & 1.00 & 133.16 & 37.60 & 8.92 & 0.86 & 0.74 \\
\hline \multirow{3}{*}{2} & BNN & 0.19 & 59.75 & 43.39 & 11.54 & 0.87 & 0.75 \\
\hline & $\overline{\text { NHHSMM }}$ & 1.00 & 132.58 & 46.65 & 13.38 & 0.66 & 0.75 \\
\hline & BLR & 1.00 & 133.44 & 40.10 & 10.78 & 0.85 & 0.75 \\
\hline \multirow[t]{3}{*}{3} & BNN & 1.00 & 59.61 & 8.75 & 2.02 & 0.97 & 0.75 \\
\hline & NHHSMM & 1.00 & 127.88 & 12.89 & 3.45 & 0.92 & 0.75 \\
\hline & $\overline{\mathrm{BLR}}$ & 1.00 & 133.51 & 8.16 & 1.68 & 0.95 & 0.75 \\
\hline \multirow[t]{3}{*}{4} & $\mathrm{BNN}$ & 0.50 & 58.61 & 33.80 & 8.06 & 0.91 & 0.75 \\
\hline & NHHSMM & 1.00 & 124.10 & 36.01 & 9.47 & 0.85 & 0.75 \\
\hline & $\overline{\mathrm{BLR}}$ & 1.00 & 132.47 & 32.33 & 8.21 & 0.86 & 0.75 \\
\hline \multirow[t]{3}{*}{5} & $\mathrm{BNN}$ & 1.00 & 69.48 & 10.54 & 2.39 & 0.95 & 0.75 \\
\hline & NHHSMM & 1.00 & 130.03 & 9.05 & 2.23 & 0.88 & 0.75 \\
\hline & BLR & 1.00 & 133.53 & 10.83 & 2.36 & 0.93 & 0.75 \\
\hline \multirow[t]{3}{*}{6} & $\mathrm{BNN}$ & 1.00 & 78.10 & 6.37 & 1.35 & 0.97 & 0.74 \\
\hline & NHHSMM & 0.98 & 128.09 & 15.05 & 3.09 & 0.93 & 0.74 \\
\hline & $\overline{\mathrm{BLR}}$ & 1.00 & 132.65 & 14.76 & 3.61 & 0.90 & 0.74 \\
\hline \multirow[t]{3}{*}{7} & $\mathrm{BNN}$ & 1.00 & 70.18 & 10.56 & 2.67 & 0.95 & 0.74 \\
\hline & NHHSMM & 1.00 & 129.05 & 20.14 & 4.74 & 0.89 & 0.74 \\
\hline & $\overline{\mathrm{BLR}}$ & 1.00 & 133.62 & 13.50 & 3.66 & 0.93 & 0.74 \\
\hline \multirow[t]{3}{*}{8} & BNN & 1.00 & 59.06 & 8.46 & 1.98 & 0.95 & 0.75 \\
\hline & NHHSMM & 1.00 & 128.07 & 6.16 & 1.37 & 0.94 & 0.75 \\
\hline & BLR & 1.00 & 133.50 & 6.72 & 1.56 & 0.94 & 0.75 \\
\hline \multirow[t]{3}{*}{9} & BNN & 1.00 & 118.17 & 19.65 & 6.90 & 0.87 & 0.75 \\
\hline & $\overline{\text { NHHSMM }}$ & 0.33 & 77.78 & 36.24 & 14.62 & 0.77 & 0.75 \\
\hline & BLR & 0.84 & 135.14 & 52.92 & 20.12 & 0.71 & 0.33 \\
\hline \multirow[t]{3}{*}{10} & BNN & 1.00 & 85.84 & 17.11 & 5.27 & 0.93 & 0.75 \\
\hline & NHHSMM & 0.35 & 110.65 & 45.79 & 13.71 & 0.75 & 0.15 \\
\hline & BLR & 1.00 & 133.44 & 9.19 & 2.74 & 0.95 & 0.75 \\
\hline \multirow[t]{3}{*}{11} & $\mathrm{BNN}$ & 0.99 & 154.91 & 37.81 & 7.44 & 0.88 & 0.75 \\
\hline & NHHSMM & 0.98 & 162.43 & 46.43 & 9.89 & 0.69 & 0.75 \\
\hline & BLR & 0.80 & 132.20 & 58.38 & 12.00 & 0.80 & 0.68 \\
\hline \multicolumn{8}{|c|}{ Average Metrics } \\
\hline & $\mathrm{BNN}$ & 0.83 & 79.46 & 21.20 & 5.27 & 0.92 & 0.75 \\
\hline & NHHSMM & 0.84 & 125.10 & 29.27 & 7.91 & 0.83 & 0.69 \\
\hline & BLR & $\begin{array}{l}0.97 \\
\end{array}$ & 133.33 & 25.86 & 6.87 & 0.88 & 0.70 \\
\hline
\end{tabular}

Concerning the CICP and MCIW metrics, which quantify the coverage of the ground truth RUL from the confidence interval and the confidence interval's mean width, respectively, BLR seems to have the highest coverage probability but at the same time the widest Confidence Intervals. BNN has by far the tightest Confidence Intervals at the expense of coverage probability. NHHSMM has a slightly higher CICP value from BNN and slightly lower MCIW value than BLR. Optimizing both those metrics, by acquiring minimum MCIW and maximum CICP, results in an optimum confidence interval.
In terms of the Prognostic Horizon metric, BNN hits the absolute best (0.75) outperforming the other two algorithms. Furthermore, BNN outperforms the other two algorithms in almost every single case, when it comes to the CRA metric.

\section{CONCLUSION}

We investigated the potential of probabilistic data-driven methodologies based on statistical and AI models on the prediction of the RUL of an actual aircraft system (brakes) that are currently maintained under the TBM philosophy. For this purpose, we utilized a blind real-life dataset generated by a wide-body aircraft from a large European Airliner. A rather simple data preprocessing through moving average filtering was followed and produced quite good degradation histories. We utilized three state-of-the-art mathematical models to implement the prognostic task with the results being summarized in Figures 5, $6 \& 7$.

The main conclusion is the feasibility of all models to successfully predict the Remaining Useful Life of the aircraft brakes even from very early on during their service time. For the best performing model i.e., BNNs, mean predictions of $<10 \%$ MAPE are achieved, all across the testset and even for outlier cases. This is an important achievement towards a Condition-Based-Maintenance paradigm shift in commercial aviation. It was successfully demonstrated that aircraft brakes' useful safe operation can be reliably prognosed through a data-driven approach that relies on available historical data without requiring extremely sophisticated or computationally intensive algorithms. BLR and NHHSMM perform very well in most test-set cases but rather underperform in the outliers' scenarios. Also, well established models such as BNNs and BLRs outperformed a more sophisticated approach such as the NHHSMM. This is an interesting conclusion that highlights the importance of a careful selection of the mathematical model that is to be utilized for the prognostic methodology development.

Finally, it becomes apparent, that in order to achieve the prognostic task even in a problem with strong linear correlation of sensor data to remaining useful life one has to resort to advanced algorithms. Even though the correlation is linear for each brake, the slope of each degradation curve is rather stochastic (see Figure 2b). If a simple linear regression method was utilized, a deterministic curve with a single slope would result, in order to estimate the Remaining Useful Life of the aircraft brakes. Besides this approach would severely underperform in outlier cases it cannot moreover quantify the uncertainty behind the mean prediction, an aspect of utmost importance from a maintenance planning point of view. To capture (at least partially) outlier cases you need to quantify the uncertainty behind the mean estimate and determine confidence intervals. Thus, more sophisticated mathematics are required i.e., probabilistic versions of "simple" algorithms etc. Interestingly enough, the Bayesian version of the "simple" 
linear regression is not the best performer as we demonstrated in the paper. The transition to CBM of aircraft systems fundamentally calls for reliable prognostics. The present work demonstrates that this is feasible but the road towards a Condition-Based-Maintenance paradigm shift in commercial aviation has still several challenges ahead that are beyond the objectives of the present work.

\section{ACKNOWLEDGEMENT}

The present work was financially supported by the European Union's Horizon 2020 research and innovation programme ReMAP (Grant Agreement Number: 769288). The support is sincerely appreciated by the authors.

\section{REFERENCES}

Acuna, D. E. \& Orchard, M. E. (2017). Particle-filteringbased failure prognosis via sigma-points: Application to Lithium-Ion battery state-of-charge monitoring, Mechanical Systems and Signal Processing, 85, pp. 827-848, https://doi.org/10.1016/j.mssp.2016.08.029

Adhikari, P.P. \& Buderath, M. A framework for aircraft maintenance strategy including CBM, Proceedings of the European Conference Prognostics Health Management Society 2016, pp. 1-10.

Autin, S.; De Martin, A.; Jacazio, G.; Socheleau, J.; Vachtsevanos, G. (2021), International Journal of Prognostics and Health Management, Results of a Feasibility Study of a Prognostic System for ElectroHydraulic Flight Control Actuators, 12 (3), pp. 1-18. https://doi.org/10.36001/ijphm.2021.v12i3.2935

Che, C.; Wang, H.; Fu, Q.; Ni, X. (2019) Combining multiple deep learning algorithms for prognostic and health management of aircraft, Aerospace Science and Technology, 94, 105423. https://doi.org/10.1016/j.ast.2019.105423

Dalla Vedova, M.D.L.; Germanà, A.; Berri, P.C.; Maggiore, P. (2019). Model-Based Fault Detection and Identification for Prognostics of Electromechanical Actuators Using Genetic Algorithms. Aerospace 6 (94) https://doi.org/10.3390/aerospace6090094

Dawn, A,; Kim, N.H.; Choi, J-H. (2015) Practical options for selecting data-driven or physics-based prognostics algorithms with reviews, Reliability Engineering \& System Safety, 133, pp. 223-236. https://doi.org/10.1016/j.ress.2014.09.014

Efron, B.; Tibshirani, R.J. (1993) An Introduction to the Bootstrap, Chapman and Hall, New York, https://doi.org/10.1007/978-1-4899-4541-9

Eleftheroglou, N.; Mansouri, S.S.; Loutas, T.; Karvelis, P.; Georgoulas, G.; Nikolakopoulos, G.; Zarouchas, D. (2019). Intelligent data-driven prognostic methodologies for the real-time remaining useful life until the end-of-discharge estimation of the LithiumPolymer batteries of unmanned aerial vehicles with uncertainty quantification, Applied Energy, 254,
113677.

https://doi.org/10.1016/j.apenergy.2019.113677

Eleftheroglou, N.; Zarouchas, D.; Loutas, T.; Alderliesten, R.; Benedictus, R. (2018). Structural health monitoring data fusion for in-situ life prognosis of composite structures, Reliability Engineering \& System Safety, 178, pp. 40-54. https://doi.org/10.1016/j.ress.2018.04.031

El-Sayed, M.; Riad, F.; Elsafty, M.; Estaitia, Y. (2017). Algorithms of Confidence Intervals of WG Distribution Based on Progressive Type-II Censoring Samples. Journal of Computer and Communications, 5, pp. 101116. https://doi: 10.4236/jcc.2017.57011.

Ezhilarasu, C.M.; Skaf, Z.; Jennions, I.K. (2019). The application of reasoning to aerospace Integrated Vehicle Health Management (IVHM): Challenges and opportunities, Progress in Aerospace Sciences, 105 pp. 60-73, https://doi.org/10.1016/j.paerosci.2019.01.001

Goebel, K.; Daigle, M.; Saxena, A.; Sankararaman, S.; Roychoudhury, I.; Celaya, (2017), Prognostics: The science of prediction, CA, CreateSpace Independent Publishing Platform; 1st ed.

Jia, X.; Huang, B.; Feng, J.; Cai, H.; Lee, J. (2018). A Review of PHM Data Competitions from 2008 to 2017: Methodologies and Analytics. Proceedings of the Annual Conference of the Prognostics and Health Management Society, Philadelphia, Pennsylvania, USA.

Kallen, M.J. \& van Noortwijk, J.M. (2005) Optimal maintenance decisions under imperfect inspection, Reliability Engineering and System Safety, 90 (2-3), pp. 177-185. https://doi.org/10.1016/j.ress.2004.10.004

Khosravi, A., Nahavandi, S., Creighton, D. and Atiya, A. F. (2011). Comprehensive Review of Neural NetworkBased Prediction Intervals and New Advances, IEEE Transactions on Neural Networks, 22 (9) pp. 13411356, doi: 0.1109/TNN.2011.2162110.

Lee, J. \& Mitici, M. (2020). An integrated assessment of safety and efficiency of aircraft maintenance strategies using agent-based modelling and stochastic Petri nets, Reliability Engineering \& System Safety, 202, 107052. https://doi.org/10.1016/j.ress.2020.107052

Li, R.; Verhagen, W.J.C.; Curran, R. (2020) Toward a methodology of requirements definition for prognostics and health management system to support aircraft predictive maintenance, Aerospace Science and Technology, 102, 105877. https://doi.org/10.1016/j.ast.2020.105877

Loutas, T.; Eleftheroglou, N.; Zarouchas, D. (2017) A datadriven probabilistic framework towards the in-situ prognostics of fatigue life of composites based on acoustic emission data, Composite Structures, 161, pp. 522-529.

https://doi.org/10.1016/j.compstruct.2020.112386

Loutas, T.; Eleftheroglou, N.; Georgoulas, G.; Loukopoulos, P.; Mba D.; Bennett, I. (2020). Valve Failure Prognostics in Reciprocating Compressors Utilizing 
Temperature Measurements, PCA-Based Data Fusion, and Probabilistic Algorithms, IEEE Transactions on Industrial Electronics, 67 (6), pp. 5022-5029, doi: 10.1109/TIE.2019.2926048.

Lu, F.; Wu, J.; Huang, J.; Qiu, X. (2019). Aircraft engine degradation prognostics based on logistic regression and novel OS-ELM algorithm, Aerospace Science and Technology, 84, pp. 661-671. https://doi.org/10.1016/j.ast.2018.09.044

Moghaddass, R.; Zuo, M. J. (2014). An integrated framework for online diagnostic and prognostic health monitoring using a multistate deterioration process, Reliability Engineering \& System Safety, 124, pp. 92104. https://doi.org/10.1016/j.ress.2013.11.006

Nix, D.A.; Weigend, A.S. (1995). Learning local error bars for nonlinear regression, Advances in Neural Information Processing Systems, vol. 7, G. Tesauro, D. Touretzky, and T. Leen, Eds. Cambridge, MA, USA: MIT Press, pp. 489-496.

Pierce, S. G.; Worden, K.; Bezazi, A. (2008). Uncertainty analysis of a neural network used for fatigue lifetime prediction, Mechanical Systems Signal Processing, 22 (6), pp. 1395-1411. https://doi.org/10.1016/j.ymssp.2007.12.004

Rengasamy, D.; Jafari, M.; Rothwell, B.; Chen X.; Figueredo, G. (2020). Deep Learning with Dynamically Weighted Loss Function for Sensor-Based Prognostics and Health Management, Sensors, 20 (3), 723; https://doi.org/10.3390/s20030723

Sankararaman, S. \& Goebel, K. (2020) Uncertainty in prognostics and systems health management, International journal of prognostics and health management, $\quad$ pp.1-14 https://doi.org/10.36001/ijphm.2015.v6i4.2319.

Strategic Research \& Innovation Agenda, Vol. 2, Advisory Council for Aviation Research and Innovation in Europe (ACARE), September 2012, www.acare4europe.com

Saxena A, Celaya J, Saha B, Saha S, Goebel K. (2020) Metrics for offline evaluation of prognostic performance, International. Journal Prognostics Health Management, 1, pp.1-20.

Tipping, M. Sparse Bayesian learning and the relevance vector machine, Journal of machine learning research 1, 2001, pp. 211-244.

Verstraete, D.; Droguett, E.; Modarres, M. A Deep Adversarial Approach Based on Multi-Sensor Fusion for Semi-Supervised Remaining Useful Life Prognostics, Sensors 2020, 20(1), 176; https://doi.org/10.3390/s20010176 